This is a repository copy of A Coalitional Model Predictive Control for the Energy Efficiency of Next-Generation Cellular Networks in the Depósito de Investigación de la Universidad de Sevilla

Version: Author Accepted Version

Citation: A Coalitional Model Predictive Control for the Energy Efficiency of NextGeneration Cellular Networks. Eva Masero, Luis A. Fletscher, José M. Maestre. Energies. Vol. 13-24. 10.23919/ecc51009.2020.9143643

To cite this publication, please use the final published version (if applicable). Please check the document version above.

Copyright: Other than for strictly personal use, it is not permitted to download, forward or distribute the text or part of it, without the consent of the author(s) and/or copyright holder(s), unless the work is under an open content license such as Creative Commons.

Takedown policy: Please contact us (idus@us.es) and provide details if you believe this document breaches copyrights. We will remove access to the work immediately and investigate your claim 


\title{
A Coalitional Model Predictive Control Approach for Heterogeneous Cellular Networks*
}

\author{
Eva Masero ${ }^{1}$, Luis A. Fletscher ${ }^{2}$, and José M. Maestre ${ }^{1}$.
}

\begin{abstract}
Heterogeneous cellular networks (HetNets) are large-scale systems that comprise numerous base stations interacting with a significant number of users of diverse types. Finding a trade-off between energy consumption and quality of service is one of the major challenges in these networks. To deal with this issue, a coalitional model predictive control (MPC) approach is proposed for a HetNet powered by renewable power sources, and compared in simulation with the traditional best-signal level mechanism and the centralized MPC method. Furthermore, other key performance indicators associated with grid consumption such as the number of served users and transmission rates are also evaluated.
\end{abstract}

\section{INTRODUCTION}

Large-scale systems are formed by multiple subsystems that inter-operate to reach a common global objective and are distributed over a wide geographical area, e.g., power systems. These subsystems may be of diverse nature as happens in heterogeneous cellular networks (HetNets) [1], which are composed of high-power macro-base stations and low-power small-cell base stations (see Fig. 1). One of the main challenges related to the control of large-scale cellular networks is the coordination of user flows due to the coupling between subsystems. Another complicating issue is that centralized control methods are extremely complex or even impossible to implement in such large-scale systems.

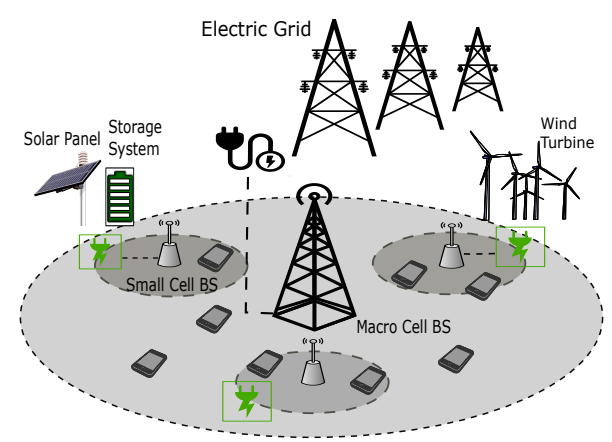

Fig. 1. Scenario: A HetNet powered by hybrid energy sources.

\footnotetext{
*This work was supported by the Colombian funding entity Departamento Administrativo de Ciencia, Tecnología e Innovación - COLCIENCIAS for the Ph.D. scholarship number 6172; the Spanish MINECO project DPI201786918-R and the H2020 ADG-ERC project OCONTSOLAR (ref. 789051); and the Spanish MICINN for the Ph.D. grant number FPU18/04476.

1 Eva Masero and José M. Maestre are with Departamento de Ingeniería de Sistemas y Automática, Escuela Técnica Superior de Ingeniería, Universidad de Sevilla, Sevilla, Spain evamaserodus.es, pepemaestredus.es

${ }^{2}$ L. Fletscher is with Departamento de Ingeniería Electrónica y Telecomunicaciones, Facultad de Ingeniería, Universidad de Antioquia UdeA, Medellín, Colombia luis.fletschereudea.edu.co
}

In this work, we are interested in the model predictive control (MPC) strategy [2], which is based on the receding horizon principle. At each time step, it uses an explicit model of the system to solve a finite horizon optimization problem subject to certain constraints to calculate the optimal control input. For instance, in [3], MPC is applied to manage the power signal in the base stations (BSs) and reduce the negative impact of disturbances in the transmission process. Likewise, the reduction of co-channel interference through distributed power adaptation using MPC is addressed in [4].

However, centralized MPC demands a high computation burden that it is hard to provide for the problem considered. For that reason, many distributed MPC (DMPC) approaches have been proposed to deal with large-scale problems over the last decades [5]. The key idea is to divide the system into subsystems governed by individual controllers (agents) to achieve a common global objective. The distributed problem can be formulated following two different approaches: i) a non-cooperative strategy where agents solve their problems considering only their local interests [6], and ii) a cooperative scheme where agents bear in mind other agents interests as well to obtain better global performance [7]. A halfway strategy is to consider a dynamic partitioning of the system so as to achieve a balance between coordination overheads and local performance, which is the rationale of the recently proposed coalitional MPC [8], [9].

The main contribution of this paper is the assessment of coalitional MPC in cellular networks powered by hybrid energy sources. Indeed, various studies have shown that using renewable energy sources in HetNets reduces network costs [10]-[12] and improves environmental factors [13]. Another appealing feature is the possibility of deploying infrastructure in off-grid and connection limited scenarios (e.g., in developing countries and disaster-affected areas). Hence, renewable energies might be a complementary element in next-generation cellular networks (NGCN) to make these systems more self-sustainable [14]. Nevertheless, the integration of renewable energies in NGCN presents various challenges related to the management of the network architecture and the adaptation to the changing energy source behavior [15], which will put coalitional MPC to the test. Additionally, the proposed control strategy is compared with the traditional best-signal-level mechanism and centralized MPC in simulations based on real data.

The outline of rest of the article is as follows. Section II states the problem. Section III details user-BS association mechanisms focusing on the coalitional MPC scheme. In Section IV, the performance of the proposed strategy is 
evaluated in simulation. Finally, conclusions are provided in Section V.

\section{Problem Statement}

Let us consider a two-tier downlink HetNet as shown in Fig. 1, which is composed of a set of $\mathcal{B}=\{1,2, \ldots, B\}$ subsystems: one macro-base station (MBS) and multiple small-cell base stations (SCBSs). Specifically, the MBS is always available and powered by on-grid energy, whilst the SCBSs are exclusively powered by renewable energy and also equipped with a battery system. Although the MBS provides basic coverage, SCBSs are deployed to enhance network capacity and receive traffic from the MBS.

The discrete-time dynamics of each base station $i \in \mathcal{B}$ are given by

$$
x_{i}(k+1)=A_{i} x_{i}(k)+\sum_{j \in C_{i}(k)} B_{i j} u_{i j}(k)+w_{i}(k),
$$

where $k \in \mathbb{N}_{0}$ indicates the time instant; the state $x_{i} \in \mathbb{R}^{2}$ is composed by the number of users being served and the battery level of BS $i$; inputs $u_{i j} \in \mathbb{R}$ are the user flows from the set of subsystems cooperating with BS $i$, namely, $C_{i}$. Thus, it holds that $u_{i j}=-u_{j i}$ if $i, j \in C_{i}$, and $u_{i j}=0$ for all $j \notin C_{i}$. The disturbance term $w_{i} \in \mathbb{R}^{2}$ represents user-generated traffic and weather conditions that affect the generation of renewable energy. Moreover, $A_{i}$ is the state matrix and $B_{i j}$ is the input-to-state matrix from neighboring base station $j \in C_{i}$.

Controllers can cooperate through a network whose topology is described by a graph $(\mathcal{B}, \mathcal{L})$, where $\mathcal{L}$ is the set of links or edges $\mathcal{L} \subseteq \mathcal{L}^{\mathcal{B}}=\{\{i, j\} \mid\{i, j\} \subseteq \mathcal{B}, i \neq$ $j\}$. At time instant $k$, the number of active cooperation links $L \leq|\mathcal{L}|$ defines the network topology $\Lambda \in \mathcal{T} \triangleq$ $\left\{\Lambda_{0}, \Lambda_{1}, \ldots, \Lambda_{2|\mathcal{L}|-1}\right\}^{1}$. Note that the coalition problems can be considered to be decoupled due to the MBS, which is able to handle all the users coming from the SCBSs at the expense of decreasing its quality of service. Finally, the disjoint set of coalitions generated by topology $\Lambda$ is denoted by $\mathcal{B} / \Lambda$, with $\bigcup_{C \in \mathcal{B} / \Lambda} C=\mathcal{B}$ and the state evolution of each coalition $C$ is modeled as

$$
x_{C}(k+1)=A_{C} x_{C}(k)+\sum_{j \in C(k)} B_{C} u_{C}(k)+w_{C}(k),
$$

where $x_{C}=\left(x_{i}\right)_{i \in C}$ and $u_{C}=\left(u_{i j}\right)_{i, j \in C}$ are respectively the aggregate vectors of the states and inputs of the subsystems in $C$, and $w_{C}$ comprises the coupling with other coalitions and the influence of weather conditions.

\section{A. Energy Model}

The energy consumption of $\mathrm{BS} i \in \mathcal{B}$ is composed of dynamic power consumption and static power consumption as [16]

$$
E_{i}(k)=\Delta_{i} \delta_{i}(k) T_{i}+E_{i}^{\mathrm{S}}, \forall i \in \mathcal{B},
$$

where $\Delta_{i}$ is the slope of the load-dependent energy consumption; $\delta_{i}$ and $T_{i}$ are, respectively, the traffic load and the

\footnotetext{
${ }^{1}$ If the number of links is large, it is possible to consider just a subset of topologies to avoid combinatorial explosion issues.
}

transmission power; and $E_{i}^{\mathrm{S}}$ is the static energy consumption. The latter is related to the energy required for the normal operation of a BS, while the dynamic power consumption is the additional energy demand caused by the traffic load.

Since the total energy consumption is the sum of the grid consumption of the MBS $(i=1)$ and the green consumption of all SCBSs $i \in \mathcal{B} \backslash\{1\}$, the decrease in MBS consumption is essential to increase energy efficiency.

Finally, at time instant $k$, the green energy $E_{i}^{\mathrm{G}}$ available in SCBS $i$ is

$$
E_{i}^{\mathrm{G}}(k)=E_{i}^{\mathrm{G}}(k-1)-E_{i}(k-1)+\varphi(k),
$$

with $E_{i}^{\mathrm{G}}(k-1)$ being the stored green energy, $E_{i}(k-1)$ the green energy consumed at the previous step, and $\varphi(k)$ the green energy generation rate.

\section{B. Traffic Model}

Let us define a geographical area $\mathcal{A} \subset \mathbb{R}^{2}$ where base stations are located at positions $p \in \mathcal{A}$, and a set of available base stations $\mathcal{B}_{z}(k) \subseteq \mathcal{B}$ that provides service to user $z \in$ $\mathcal{Z} \triangleq\{1,2, \ldots, Z\}$ at time instant $k$. Let us also consider the spatial variability of traffic requests as an inhomogeneous Poisson point process as in [17]. Furthermore, the traffic size, the arrival rate per area $\lambda^{p}$, and the average traffic size $\mu^{p}$ are independently distributed for all $p \in \mathcal{A}$.

Given a mobile user $z \in \mathcal{Z}$, its transmission rate, denoted by $r_{z i}^{p}$, can be generally expressed according to the ShannonHartley theorem [17] as

$$
r_{z i}^{p}(k)=W_{i} \log _{2}\left(1+\psi_{z i}^{p}(k)\right), \forall z \in \mathcal{Z}, i \in \mathcal{B}_{z}(k), p \in \mathcal{A},(5)
$$

where $W_{i}$ is the operating bandwidth for all $i \in \mathcal{B}_{z}(k)$ and $\psi_{z i}^{p}$ is the received signal at location $p$ from BS $i$, which is given by the signal-to-interference-plus-noise ratio (SINR) and calculated as

$$
\psi_{z i}^{p}=\frac{T_{i} g_{i}^{p}}{\sigma^{2}+\sum_{j \in \mathcal{B}_{z} \backslash\{i\}} T_{j} g_{j}^{p}}, \forall i, j \in \mathcal{B}_{z}(k),
$$

where we recall that $T_{i} \in \mathbb{R}$ is the transmission power of BS $i$; the channel gain between the $i$-th BS and the user at position $p$ is $g_{i}^{p}$, which reflects the slow fading including the path loss and the shadowing; and $\sigma^{2}$ is the noise power level. Note that the denominator in (6) represents the interfering BSs transmission towards user $z$ at location $p$. Additionally, $\psi_{z i}^{p}$ must be higher than a threshold so that user $z$ has enough signal level.

Moreover, it is assumed that each SCBS $i \in \mathcal{B}_{z} \backslash\{1\}$ can only serve a fixed number $z_{i}^{\max }$ of users simultaneously. As for the MBS $(i=1)$, there is no limit of users served so that the availability of the service is guaranteed for all instants. Therefore, considering that mobile users are uniformly distributed in the coverage area, the traffic load $\delta_{i}$ of BS $i$ can be expressed as

$$
\delta_{i}(k)= \begin{cases}\frac{\sum_{z \in \mathcal{Z}} y_{z i}(k)}{z_{i}^{\max }} & \forall i \in \mathcal{B}_{z}(k) \backslash\{1\} \\ \frac{\sum_{z \in \mathcal{Z}} y_{z i}(k)}{|\mathcal{Z}|} & \text { otherwise, }\end{cases}
$$


where $y_{z i}$ is the user association indicator, i.e., if user $z$ is associated with the BS $i$, then $y_{z i}=1$, and $y_{z i}=0$ otherwise. Furthermore, note that $0 \leq \delta_{i}(k) \leq 1, \forall i \in \mathcal{B}_{z}(k)$.

\section{COALitional MPC AND OTHER ASSESSED METHODS}

This section details the proposed coalitional MPC method and two other alternatives: i) centralized MPC, which provides an upper-bound of the performance of the proposed method; and ii) best-signal-level mechanism, which allows assessing improvements regarding a very popular user BSassociation strategy.

\section{A. Coalitional MPC}

The rationale of coalitional MPC is allowing time-varying cooperation [8], [9] according to the requirements of users and the energy available in SCBSs to find a balance between cooperation costs and network performance. Each enabled cooperation link in topology $\Lambda \in \mathcal{T}$ has a specific cost $c_{\text {link }}$ to discourage unnecessary cooperation between base stations. Otherwise, full cooperation would be used at each time step and issues derived from the communication/computation burden might appear, e.g., excessive time to solve the optimization problem.

The goal of each coalition $C$ is to minimize the sum of a stage cost function $l_{C}(\cdot)$ and a cooperation cost function $g_{C}(\cdot)$, which can respectively be expressed as

$$
\begin{gathered}
l_{C}(\cdot)=Q\left(x_{1}^{\mathrm{z}}(k+t+1)\right)^{2}+R \sum_{i \in C}\left(u_{i, 1}(k+t)\right)^{2}, \\
g_{C}(\cdot)=c_{\text {link }} L_{\Lambda},
\end{gathered}
$$

where $x_{1}^{\mathrm{z}}$ is the number of users being served by the MBS; $u_{i, 1}$ is the traffic flow from BS $i$ to the MBS; $Q, R$ are definite-positive constant weighting matrices that penalize respectively the number of users at the MBS and its incoming flows; and $L_{\Lambda}$ represents the number of active links of topology $\Lambda$.

The assessed scheme is based on a top-down control approach [8], where an upper-layer decides the best topology to implement according to the current disturbances every $T_{\text {up }}=10$ instants. For simplicity, five topologies are considered in simulation (see Fig. 2):

- Topology $\Lambda_{1}$ corresponds to the centralized MPC scheme where full cooperation is enabled. The number of active cooperation links is $L_{\Lambda_{1}}=24$.

- Topology $\Lambda_{2}$ refers to a network where SCBSs cannot communicate between them, but only with the MBS. The number of enabled cooperation links is $L_{\Lambda_{2}}=8$.

- Topology $\Lambda_{3}$ presents a five-SCBS central coalition with a total of $L_{\Lambda_{3}}=16$ cooperation links.

- Topology $\Lambda_{4}$ considers two coalitions and presents $L_{\Lambda_{4}}=14$ cooperation links.

- Topology $\Lambda_{5}$ maintains $L_{\Lambda_{5}}=12$ enabled cooperation links.

Once a topology $\Lambda \subseteq \mathcal{L}$ is chosen, it is sent to the lowercontrol layer where coalitions implement it. In particular, the problem solved by coalition $C \in \mathcal{B} / \Lambda$ at each time step is

$$
\min _{u_{C}} \sum_{t=0}^{N_{\mathrm{p}}-1} l_{C}\left(x_{1}^{\mathrm{z}}(k+t+1), u_{C}(k+t)\right)+g_{C}\left(L_{\Lambda}\right),
$$

subject to (2) and

$$
\begin{gathered}
\sum_{i, j \in C} u_{i, j}(k+t) \leq F_{i, j}(k+t) \quad t=0, \ldots, N_{\mathrm{p}}-1, \\
\sum_{j \in C} u_{i, j}(k)=x_{i}^{\mathrm{z}}(k) \quad i \in \mathcal{B} \quad k=0, \ldots, N_{\mathrm{p}}-1, \\
0 \leq u_{i, j}(k) \quad i, j \in \mathcal{B} \quad k=0, \ldots, N_{\mathrm{p}}-1,
\end{gathered}
$$

where constraint (10) specifies that the traffic flow between two BSs cannot exceed certain limits; (11) imposes flow conservation, i.e., the sum of traffic flows cannot exceed the load at BS $i$; and, finally, (12) defines that flows must be positive.

\section{B. Centralized MPC}

When full cooperation is enabled, problem (9) is solved for the grand coalition $C=\mathcal{B}$ at each time instant, i.e., with full system information. Hence, its sheer performance is the best. Nevertheless, this method requires full cooperation at each time step, thus leading to higher cooperation costs, which may hinder its implementation.

\section{Best-signal-level Policy}

In traditional cellular networks, mobile users connect to the BS that offers the best SINR, which depends on BS power transmission, path loss, and interference from other BSs. Nonetheless, this mechanism is not entirely adequate for HetNets because SCBSs with available resources may be ignored by users receiving a stronger signal from MBS [18]. This procedure will be referred to as traditional scheme and will be the baseline for evaluating the performance of the proposed coalitional MPC mechanism.

\section{CAse STUdy}

The coalitional control method is evaluated in a simulated environment using MATLAB $R$. The objective is to analyze its impact on grid-power consumption and stored energy management. Although the case study used in simulation is academic, it is sophisticated enough to show the potential of the proposed method. Moreover, it is representative of a real scenario where the complexity of the association process is caused by the number of BSs and active users.

As stated previously, the execution of the user-BS association algorithm is assumed to be faster than the dynamical variability of traffic on the cellular network, so that the calculations can be updated accordingly as new information becomes available. Additionally, note that changes on greenenergy arrival and the spatial variability in the behavior of active users are considered. For this reason, a simulation of fifty-time instants is sufficient to assess the behavior of the proposed approach. 


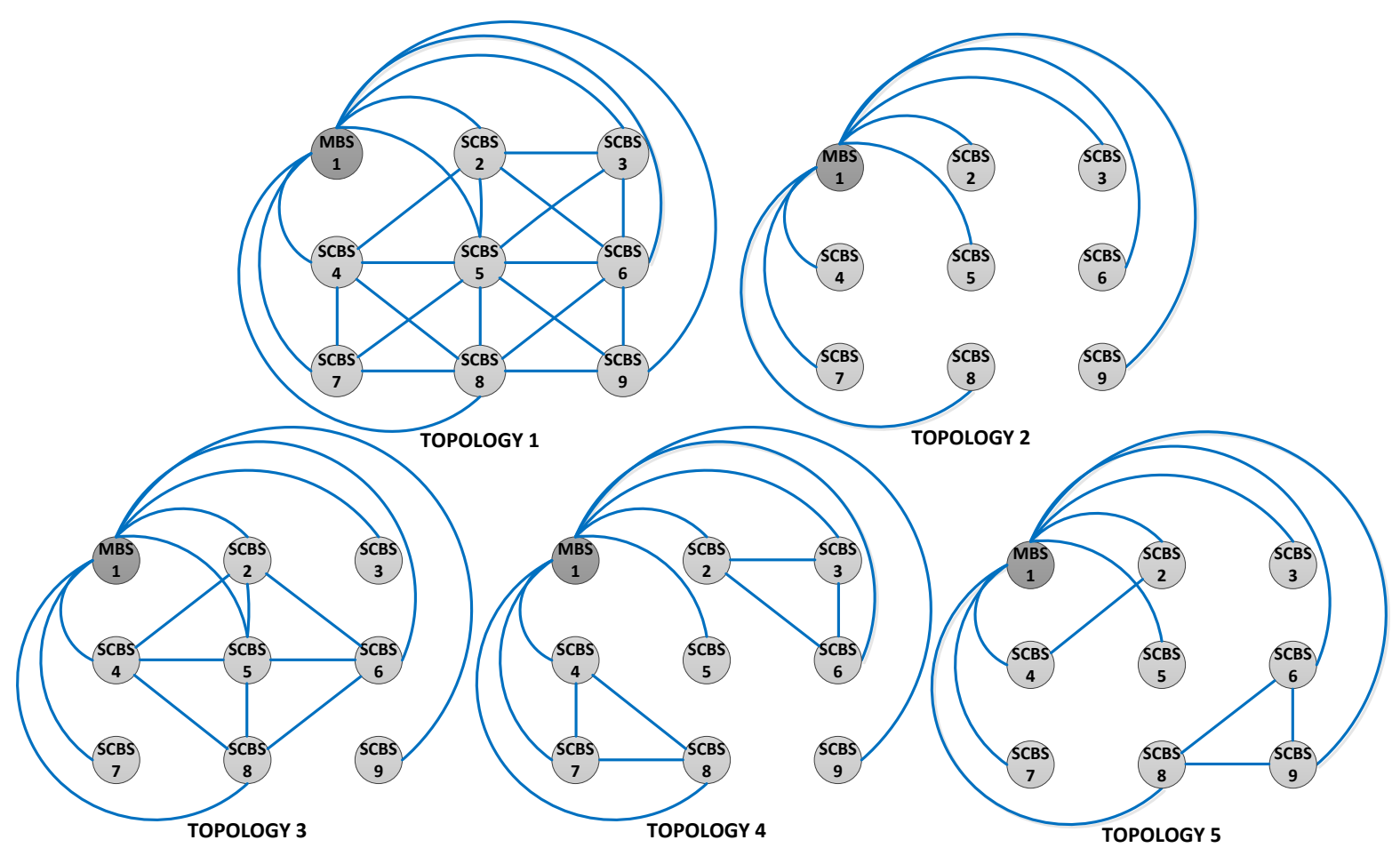

Fig. 2. Set of topologies considered in the coalitional MPC algorithm.

\section{A. Description}

The scenario described in Section II is implemented to evaluate the proposed mechanism. The system is composed of one MBS and eight overlapping SCBSs. The MBS is powered by on-grid energy, ensuring constant coverage over the area. SCBSs are powered by renewable energy and they have storage systems. Only path loss is considered between users and BSs in simulation. Moreover, user requests follow a homogenous Poisson point process where $\lambda^{p}=\lambda$ for the sake of simplicity.

From a telecommunications viewpoint, the technical parameters of the simulation are defined according to a longterm-evolution system over the area $\mathcal{A}=3.5 \mathrm{~km}^{2}$ [19]. The distance between BSs is $500 \mathrm{~m}$ and users are uniformly distributed. Table I summarizes the parameters used in simulation. According to [20], the consumption slope of MBS and SCBSs are respectively $\Delta_{1}=4.7$ and $\Delta_{i}=4.0, \forall i \in \mathcal{B} \backslash\{1\}$. Additionally, the static power consumption of the MBS and SCBSs are $E_{1}^{\mathrm{S}}=130 \mathrm{~W}$ and $E_{i}^{\mathrm{S}}=6.8 \mathrm{~W}$, respectively. The maximum transmission power of the MBS is $T_{1}=43 \mathrm{dBm}$ and $T_{i}=22 \mathrm{dBm}$ for SCBSs. Users move according to a random walk point model with an average speed of $4 \mathrm{~km} / \mathrm{h}$ [21].

To evaluate the performance of our scheme, a dynamic scenario with controlled renewable energy generation is used. Specifically, a green energy profile enables different groups of SCBSs during specific periods. Thus, active BSs change according to a pre-defined sequence while users are moving. Fig. 3 presents the arrival of green energy and users in the presented scenario. Note that user departures are also
TABLE I

SIMULATION PARAMETERS.

\begin{tabular}{lcc}
\hline \multicolumn{1}{c}{ Parameter } & Value & Units \\
\hline Coverage Area & 3.5 & $\mathrm{~km}^{2}$ \\
System & LTE & - \\
BW LTE & 20 & $\mathrm{MHz}$ \\
RB per BS & 100 & - \\
No. Macro Base Station & 1 & - \\
No. MBS sectors & 1 & - \\
No. SCBS & 8 & - \\
Inter-site distance & 500 & $\mathrm{~m}$ \\
Transmission power MBS & 43 & $\mathrm{dBm}$ \\
Transmission power SCBS & 22 & $\mathrm{dBm}$ \\
Static Power Cons. MBS & 130 & $\mathrm{~W}$ \\
Static Power Cons. SCBS & 6.8 & $\mathrm{~W}$ \\
Consumption Slope MBS & 4.7 & - \\
Consumption Slope SCBS & 4.0 & - \\
Path loss between MBS and user & Cost $231 \mathrm{model}$ & - \\
Antenna Gain & 15 & $\mathrm{dBi}$ \\
Max. users simultaneously for an SCBS & 100 & - \\
Receiver sensitivity & -107.5 & $\mathrm{dBm}$ \\
Size of request file & 500 & $\mathrm{~KB}$ \\
Time instant length & 1 & $\mathrm{~s}$ \\
Mobility Model & Random walk point & - \\
Mobility Speed & 4 & $\mathrm{~km} / \mathrm{h}$ \\
\hline
\end{tabular}

considered throughout the simulation time. This information is used by the MPC to select the best actions for grid consumption reduction.

\section{B. Energy Assessment}

To compare the performance of the coalitional MPC mechanism with the other schemes presented in Section III, 


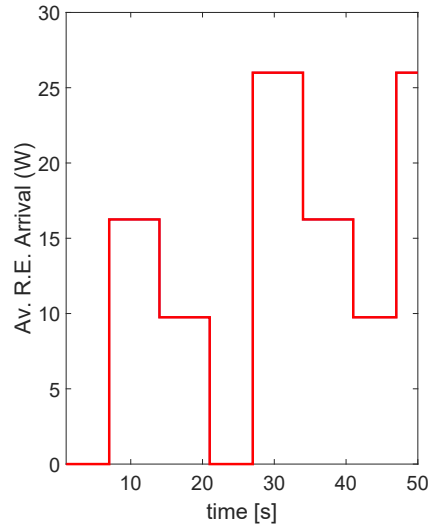

(a)

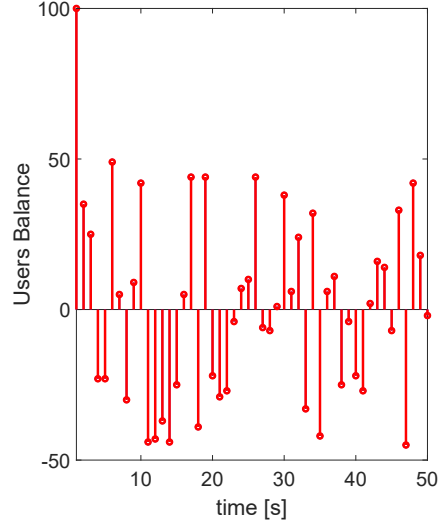

(b)
Fig. 3. (a) Average green-energy arrival. (b) Users forecast for the MBS.

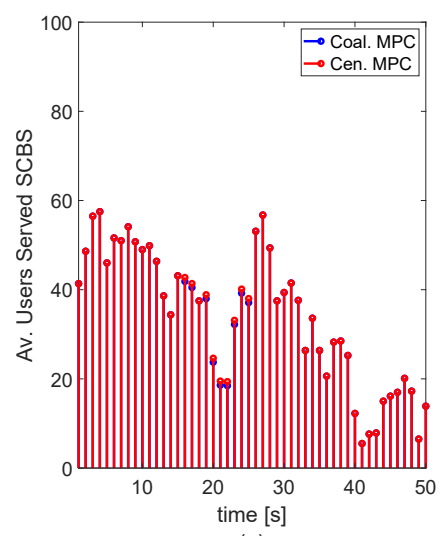

(a)

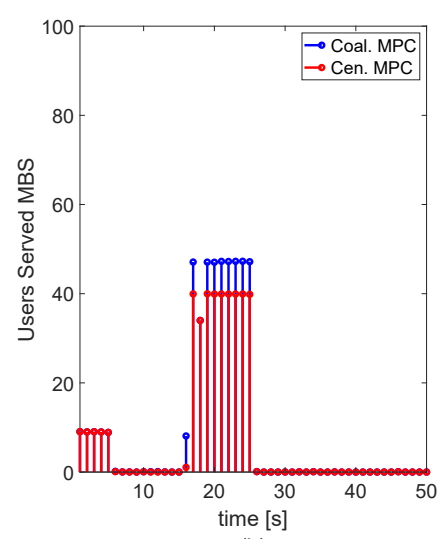

(b)
Fig. 4. (a) Average users load of SCBSs. (b) MBS users load.

five key performance indicators (KPIs) are proposed: i) grid consumption (kWatts-h); ii) the percentage of consumption reduction in comparison with the traditional scheme; iii) average stored energy (Watts); iv) performance cost; and v) cooperation cost. The simulation scenario is configured to change the number of active SCBSs according to the arrival of green energy, and it is also assumed that the batteries charge are full initially. Furthermore, the prediction horizon $N_{\mathrm{p}}$ used in these MPC mechanisms is 10 time instants.

The first parameter to evaluate is grid consumption, which is related to the number of users served by the MBS. In Fig. 4, it is observed how MPC-based mechanisms balance the traffic load, minimizing the number of users served by the MBS and increasing those served by SCBSs. In this sense, Table II shows that centralized MPC reduced grid consumption by $39.39 \%$. Coalitional MPC has a $29.54 \%$ of reduction compared with the traditional best-signal-level mechanism. These results show the effectiveness of MPCbased schemes to reduce grid consumption and increase energy efficiency.

Additionally, Table II shows stored energy management in MPC-based mechanisms. Specifically, centralized MPC achieves an average stored energy of $16.33 \mathrm{~W}$, while coalitional MPC has a stored energy average of 17.14 W. Compar- ing these results with the $27.60 \mathrm{~W}$ of the traditional scheme, it is obtained an improvement close to $40 \%$ in the stored energy indicator.

Centralized and coalitional MPC are compared in Fig. 5, where it is shown the evolution of four SCBSs. The first row shows the renewable energy arrival for each SCBS and the second row represents the expected users flow throughout the simulation time. Note that these are disturbances for the controllers, which compute suitable control actions to reduce grid consumption and manage the stored energy efficiently. The third row displays the control strategy followed by MPC schemes. An overall view of the stored energy behavior shows the response of the controller in periods without green energy arrival. For instance, for the time range between time instants 20 and 25, the number of served users by SCBSs decrease to preserve battery levels until the next green energy arrival. Note that this is consistent with the MBS behavior presented in Fig. 4 (b).

In general, both MPC-based strategies provide a similar behavior regarding stored energy management, grid consumption reduction, and traffic load of SCBSs. Even so, the coalitional proposal is more appropriate for distributed implementations.

\section{Cooperation and Performance Assessment}

Centralized MPC provides the best performance at the expense of fixed full cooperation between BSs. On the other hand, the coalitional MPC scheme varies the network topology in real-time according to changes in user traffic and weather conditions.

In the lower-control layer of the proposed coalitional scheme, the optimization problem (9) is solved by each coalition $C \in \mathcal{B} / \Lambda$ at each time instant $k$, with weighting matrices being $Q=100, R=1$, and a cost per cooperation link $c_{\text {link }}=10^{4}$. The topologies selected over the simulation are $\Lambda_{\text {hist }}=\{5,5,2,2,2\}$. Moreover, Table III displays the sum of accumulated performance (7) and cooperation (8) costs for all coalitions $C \in \mathcal{B} / \Lambda$ in both MPC schemes throughout the simulation $\left(T_{\text {sim }}=50\right.$ s $)$, i.e.,

$$
\begin{aligned}
\mathbf{J}_{\text {perf }} & =\sum_{k=0}^{T_{\text {sim }}} \sum_{C \in \mathcal{B} / \Lambda} l_{C}(\cdot), \\
\mathbf{J}_{\text {coop }} & =\sum_{k=0}^{T_{\text {sim }}} \sum_{C \in \mathcal{B} / \Lambda} g_{C}(\cdot) .
\end{aligned}
$$

TABLE III

COMPARATIVE OF COSTS IN BOTH MPC-BASED STRATEGIES.

\begin{tabular}{ccc}
\hline Accumulated Cost & Centralized MPC & Coalitional MPC \\
\hline $\mathbf{J}_{\text {perf }}$ & $1.5209 \cdot 10^{6}$ & $2.0746 \cdot 10^{6}$ \\
$\mathbf{J}_{\text {coop }}$ & $1.25 \cdot 10^{7}$ & $0.44 \cdot 10^{7}$ \\
$\mathbf{J}_{\text {total }}=\mathbf{J}_{\text {perf }}+\mathbf{J}_{\text {coop }}$ & $1.4021 \cdot 10^{7}$ & $6.4746 \cdot 10^{6}$ \\
\hline
\end{tabular}

Note that centralized MPC has a $26.68 \%$ better performance cost than coalitional MPC. However, the latter presents a cost reduction of $53.82 \%$ regarding centralized MPC when cooperation costs are also taken into account. 
TABLE II

SCHEMES COMPARISON IN FULLY-DyNAMIC SCENARIO.

\begin{tabular}{cccc}
\hline Association Scheme & Grid Consumption (kWatts-h) & Percentage of Reduction in Grid Consumption (\%) & Average Stored Energy (W) \\
\hline Best-signal-level & 396.12 & - & 27.60 \\
Centralized MPC & 240.06 & 39.39 & 16.33 \\
Coalitional MPC & 279.10 & 29.54 & 17.14 \\
\hline
\end{tabular}
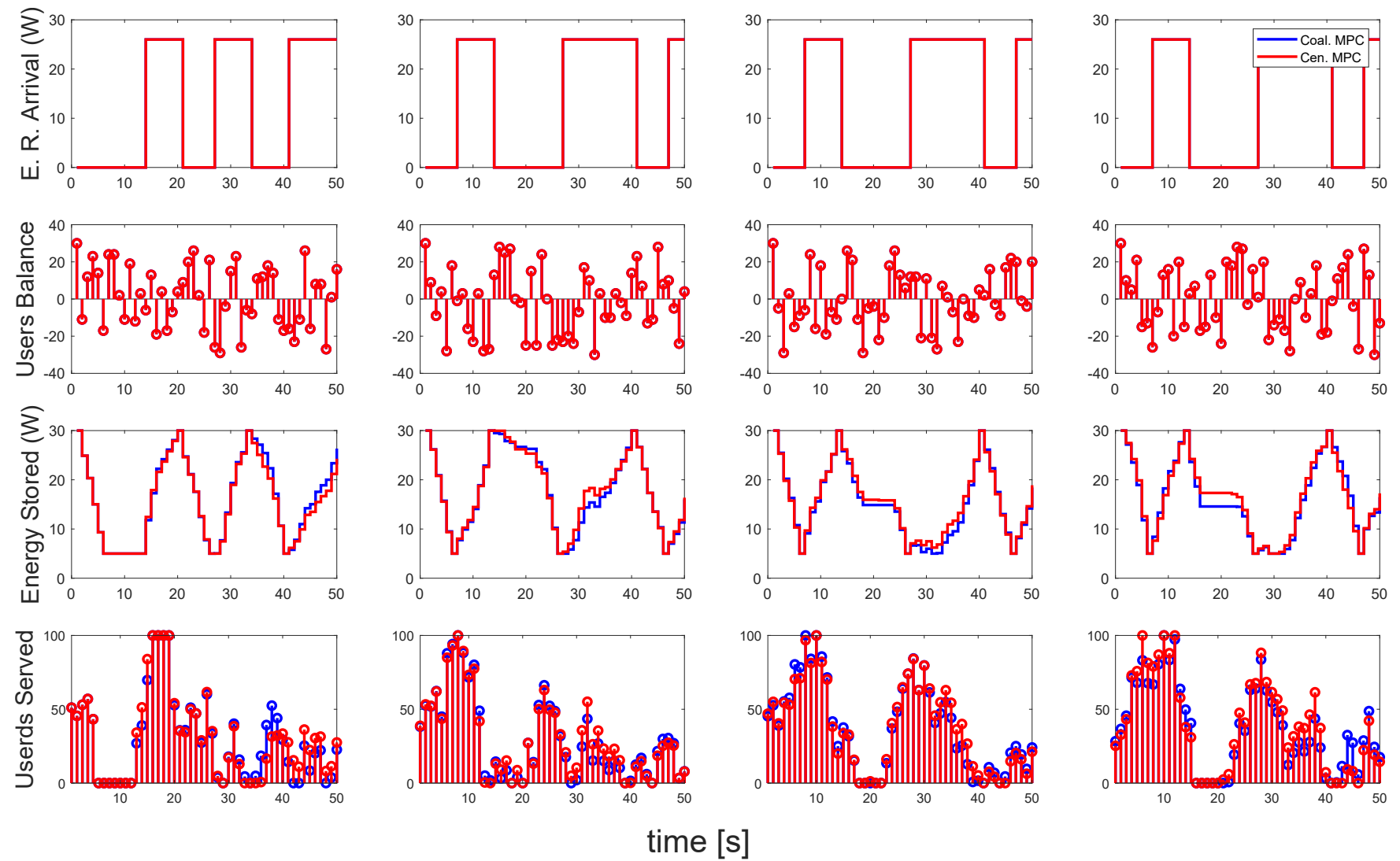

Fig. 5. Performance comparison between centralized MPC and the proposed coalitional MPC scheme.

\section{User Experience Assessment}

Traditional schemes based on signal level typically provide users with better average transmission rates than those based on another criterion for user-BS association [22]. Therefore, the evaluation of the impact of the proposed mechanism on the user experience is significant.

The percentage of users served and the average transmission rate are the KPIs used to assess user experience. In particular, the former KPI in MPC-based schemes is higher than in the best-signal level strategy, as displayed in Table IV. Regarding average transmission rates, MPC-based mechanisms have reductions close to $4 \%$ in comparison to the traditional scheme. As stated previously, these results stem from the relationship between the signal level and the user rate, being an expected outcome. Conversely, note that the coalitional MPC scheme maintains the level obtained by centralized MPC. This result, jointly with other advantages presented in previous subsections, shows that coalitional MPC is a suitable option for efficient energy management in cellular networks.
TABLE IV

EVALUATION OF USER EXPERIENCE.

\begin{tabular}{ccc}
\hline Mechanism & Users Served (\%) & Transm. Rate Reduct. (\%) \\
\hline Best-Signal Level & 95.6 & - \\
Centralized MPC & 98.1 & 4.01 \\
Coalitional MPC & 98.3 & 3.95 \\
\hline
\end{tabular}

\section{COnClusions}

An energy efficient coalitional MPC mechanism control the user-BS association process in a HetNet network powered by renewable power sources has been studied. The proposed mechanism becomes a suitable option to reduce grid consumption, discharging user flows from a grid powered MBS to green SCBSs. Furthermore, the stored energy management with coalitional MPC outperforms the traditional best-signal level mechanism while maintaining proper service levels and preserves the excellent performance of centralized MPC with a lower cooperation burden and more scalability. 
Future work will deal with a fully distributed implementation of the coalitional scheme, which is hierarchical in its current form. Furthermore, new linear models and stochastic formulations that capture the problem dynamics will be explored. Finally, implementations of these methods in a larger network and a real benchmark will also be considered.

\section{REFERENCES}

[1] A. Ghosh, N. Mangalvedhe, R. Ratasuk, B. Mondal, M. Cudak, E. Visotsky, T. A. Thomas, J. G. Andrews, P. Xia, H. S. Jo et al., "Heterogeneous cellular networks: From theory to practice," IEEE communications magazine, vol. 50, no. 6, pp. 54-64, 2012.

[2] E. F. Camacho and C. Bordons, Model predictive control. Springer Verlag London Limited, 2007.

[3] M. Cea, G. Goodwin, and T. Wigren, "Model Predictive Zooming Power Control in Future Cellular Systems under Coarse Quantization," in Proceedings of the IEEE 75th Vehicular Technology Conference (VTC Fall)), 2012.

[4] V. Garcia, N. Lebedev, and J.-M. Gorce, "Model predictive control for smooth distributed power adaptation," in 2012 IEEE Wireless Communications and Networking Conference (WCNC), 2012.

[5] J. M. Maestre and R. R. Negenborn, Distributed model predictive control made easy. Springer, 2014, vol. 69.

[6] M. Farina and R. Scattolini, "Distributed non-cooperative mpc with neighbor-to-neighbor communication," IFAC Proceedings Volumes, vol. 44, no. 1, pp. 404-409, 2011.

[7] B. T. Stewart, A. N. Venkat, J. B. Rawlings, S. J. Wright, and G. Pannocchia, "Cooperative distributed model predictive control," Systems \& Control Letters, vol. 59, no. 8, pp. 460-469, 2010.

[8] F. Fele, J. M. Maestre, and E. F. Camacho, "Coalitional Control: Cooperative Game Theory and Control," IEEE Control Systems, vol. 37, no. 1, pp. 53-69, 2017.

[9] F. Fele, E. Debada, J. M. Maestre, and E. F. Camacho, "Coalitional Control for Self-Organizing Agents," IEEE Transactions on Automatic Control, 2018.

[10] F. Morea, G. Viciguerra, D. Cucchi, and C. Valencia, "Life cycle cost evaluation of off-grid PV-wind hybrid power systems," in Proceedings of the IEEE 29th International Telecommunications Energy Conference, IEEE, Ed. Rome: IEEE, 2007, pp. 439-441.

[11] M. Marsan, G. Bucalo, A. Di Caro, M. Meo, and Y. Zhang, "Towards zero grid electricity networking: Powering BSs with renewable energy sources," in Communications Workshops (ICC), Proceedings of the 2013 IEEE International Conference on, Budapest, Hungary, June 2013, pp. 596-601.

[12] M. Alsharif, R. Nordin, and M. Ismail, "Energy optimization of hybrid off-grid system for remote telecommunication base station deployment in Malasya," EURASIP Journal on Wireless Communications and Networking, vol. 2015, no. 1, pp. 64-79, 2015.

[13] G. Piro, M. Miozzo, G. Forte, N. Baldo, L. Grieco, G. Boggia, and P. Dini, "Hetnets powered by renewable energy sources: Sustainable next-generation cellular networks," Internet Computing, IEEE, vol. 17, no. 1, pp. 32-39, Jan 2013.

[14] S. Guruacharya and E. Hossain, "Self-Sustainability of Energy Harvesting Systems: Concept, Analysis, and Design," IEEE Transactions on Green Communications and Networking, vol. 2, no. 1, pp. 175 192, 2017.

[15] H. Hassan, L. Nuaymi, and A. Pelov, "Classification of renewable energy scenarios and objectives for cellular networks," in IEEE International Symposium on Personal, Indoor and Mobile Radio Communications, PIMRC, London, UK, 2013, pp. 2967-2972.

[16] D. Liu, Y. Chen, K. K. Chai, T. Zhang, and M. Elkashlan, "Twodimensional optimization on user association and green energy allocation for hetnets with hybrid energy sources," IEEE Transactions on Communications, vol. 63, no. 11, pp. 4111-4124, 2015.

[17] H. Kim, G. de Veciana, X. Yang, and M. Venkatachalam, "Distributed $\alpha$-Optimal User Association and Cell Load Balancing in Wireless Networks," IEEE/ACM Transactions on Networking, vol. 20, no. 1, pp. 177-190, 2012.

[18] J. Andrews, "Seven ways that Hetnets are a cellular paradigm shift," IEEE Communications Magazine, vol. 51, no. 3, pp. 136-144, 2013.
[19] 3GPP, "LTE Evolved Universal Terrestrial Radio Access Network (EUTRAN); Self-configuring and self-optimizing network (SON) use cases and solutions," pp. 1-22, 2014, http://www.3gpp.org/dynareport/ 36902.htm.

[20] G. Auer, V. Giannini, C. Desset, I. Gódor, P. Skillermark, M. Olsson, M. Imran, D. Sabella, M. Gonzalez, O. Blume, and A. Fehske, "How much energy is needed to run a wireless network?" IEEE Wireless Communications, vol. 18, no. 5, pp. 40-49, 2011.

[21] W. Jiang, D.-S. Huang, and S. Li, "Random Walk-Based Solution to Triple Level Stochastic Point Location Problem," IEEE Transactions on Cybernetics, vol. 46, no. 6, pp. 1438-1451, 2016.

[22] L. Fletscher, C. Valencia, and J. M. Maestre, "An assessment of different user-BS association policies for green HetNets in off-grid environments," Transactions on Emerging Telecommunications Technologies, vol. 28, no. 12, pp. 1-15, 2017. 